\title{
Pair Distribution Function of One-dimensional "Hard Sphere" Fermi and Bose Systems
}

\author{
Bo-Bo Wei ${ }^{1}$ and Chen-Ning Yang ${ }^{1,2}$ \\ ${ }^{1}$ Chinese University of Hong Kong, Hong Kong ${ }^{2}$ Tsinghua University, Beijing, China
}

(Dated: September 1, 2021)

\begin{abstract}
The pair distributions of one-dimensional "hard sphere" fermion and boson systems are exactly evaluated by introducing gap variables.
\end{abstract}

PACS numbers: 05.30.Jp, 03.75.Hh, 67.85.Bc, 03.65.-w

\section{INTRODUCTION}

Recent experimental progress in one-dimensional (1D) systems renews theoretical interest in such systems. In this paper we study the pair distribution function in 1D "hard sphere" systems.

Pair distribution function in 1D Tonks gas [1] and Lieb-Liniger Bose gas 2, 3] have been studied previously, including the local correlation, $D(0)$, asymptotic properties at large $r$, and the zero temperature behavior [4, 5, 6, 7, 8, 9, 10, 11, 12, 13]. The pair distribution function for 3D hard sphere boson gas has been calculated by the authors 14]. In this work we address the problem of pair distribution function in 1D hard sphere fermion and boson systems with the diameter of the particles $a \neq 0$.

The pair distribution function is defined as

$$
D\left(r_{12}\right)=\rho^{-2}\left\langle\psi^{\dagger}\left(\mathbf{r}_{1}\right) \psi^{\dagger}\left(\mathbf{r}_{2}\right) \psi\left(\mathbf{r}_{2}\right) \psi\left(\mathbf{r}_{1}\right)\right\rangle
$$

where $\rho=N / L$ is the density. $D(r)$ is an important physical quantity measurable for many liquid systems. In this formula $\psi(\mathbf{r})$ is the annihilation operator in $\mathbf{r}$ space.

The pair distribution function $D\left(r_{12}\right)$ is also related to the diagonal elements of the reduced density matrix [15]:

$$
D\left(r_{12}\right)=\rho^{-2} \operatorname{Tr}\left[\psi\left(\mathbf{r}_{2}\right) \psi\left(\mathbf{r}_{1}\right) \rho_{N} \psi^{\dagger}\left(\mathbf{r}_{1}\right) \psi^{\dagger}\left(\mathbf{r}_{2}\right)\right],
$$

where $\rho_{N}=\Psi_{0} \Psi_{0}^{\dagger}$, and $\Psi_{0}$ is the many body wave function.

The meaning of $D(r)$ is: given a particle A at one point, the probability of finding another particle B at distance $r$ (counterclockwise) is

$$
\rho D(r) d r \text {. }
$$

It is obvious that $D(r) \rightarrow 1$ as $r \rightarrow \infty$. also

$$
\int_{0}^{L} \rho D(r) d r=N-1
$$

\section{FERMIONS WITH $a=0$}

\section{Wave Function $\Psi$ For Fermions With $a=0$}

First, we consider $N$ free fermions in a one-dimensional cyclic interval of length $L$. The momenta of these fermions are $2 \pi k / L$, where $k=-(N-1) / 2$ to $(N-1) / 2$. Here we consider the case that the number of fermions in the system is odd, $N=2 n+1$. The normalized many body wave function $\Psi\left(r_{1}, r_{2}, \cdots, r_{N}\right)$ of the system is of the form

$$
\Psi=\frac{1}{\sqrt{N !}} \operatorname{det}\left(\begin{array}{cccc}
\epsilon_{1}^{n} & \epsilon_{2}^{n} & \ldots & \epsilon_{2 n+1}^{n} \\
\epsilon_{1}^{n-1} & \epsilon_{2}^{n-1} & \ldots & \epsilon_{2 n+1}^{n-1} \\
\epsilon_{1}^{n-2} & \epsilon_{2}^{n-2} & \ldots & \epsilon_{2 n+1}^{n-2} \\
\vdots & \vdots & \ddots & \vdots \\
\epsilon_{1}^{-n} & \epsilon_{2}^{-n} & \ldots & \epsilon_{2 n+1}^{-n}
\end{array}\right)
$$

where $\epsilon_{j}=\exp \left(i \frac{2 \pi}{L} r_{j}\right) / \sqrt{L}$. After some calculations, we arrive at a compact form for the wave function,

$$
\Psi=\frac{1}{\sqrt{N !}}\left(\frac{1}{\sqrt{L}}\right)^{N}\left[2^{N(N-1) / 2}\right] \prod_{1 \leq i<j \leq N} \sin \left[\frac{\pi}{L}\left(r_{j}-r_{i}\right)\right]
$$

The pair distribution function $D(r)$ for free fermions can be exactly obtained from this many-body wave function [4]

$$
D(r)=1-\frac{\sin ^{2}\left(\frac{N \pi r}{L}\right)}{N^{2} \sin ^{2}\left(\frac{\pi r}{L}\right)} .
$$

\section{Gap Variables For Free Fermions}

In order to prepare for dealing with the $a \neq 0$ problem, we introduce gap variables in the case $a=0$ as follows. Consider a region of the $N$ body $L \times L \times \cdots \times L$ coordinate system where, modulo $L$,

$$
r_{1} \leq r_{2} \leq \cdots \leq r_{N} \leq r_{1}
$$

We shall designate this region as $R_{1}$. In this region, we introduce gap variables $\left\{g_{i}\right\}$ :

$$
g_{i}=r_{i+1}-r_{i}, \quad i=1,2,3, \ldots, 2 n+1 .
$$


Obviously $\sum g_{i}=L$. With the gap variables the many body wave function $\Psi$ is given by

$$
\begin{aligned}
\Psi= & Q\left[f\left(g_{1}\right) f\left(g_{2}\right) \cdots f\left(g_{2 n+1}\right)\right] \\
& \times\left[f\left(g_{1}+g_{2}\right) f\left(g_{2}+g_{3}\right) \cdots f\left(g_{2 n+1}+g_{1}\right)\right] \\
& \times\left[f\left(g_{1}+g_{2}+g_{3}\right) \cdots f\left(g_{2 n+1}+g_{1}+g_{2}\right)\right] \\
& \times \cdots \cdots \\
& \times\left[f\left(g_{1}+\cdots+g_{n}\right) \cdots f\left(g_{2 n+1}+g_{1}+\cdots+g_{n-1}\right)\right]
\end{aligned}
$$

where $f(g)=\sin (\pi g / L)$, and $Q$ is the normalization factor given by

$$
Q=\frac{1}{\sqrt{N !}}\left(\frac{1}{\sqrt{L}}\right)^{N}\left[2^{N(N-1) / 2}\right] .
$$

$\Psi$ has $(2 n+1) \times n$ factors in total.

The probability distributions in $R_{1}$ is

$$
|\Psi|^{2} d g_{1} d g_{2} \cdots d g_{N} \delta\left(\sum_{i=1}^{N} g_{i}-L\right) L
$$

with all $g_{i} \geq 0$. Now $R_{1}$ is only one of the $(N-1)$ ! regions of the full coordinate space. In each of these regions we have the same gap distribution as (12). Thus gap distribution probability $d P$ is

$$
d P=(N-1) ! L|\Psi|^{2} d g_{1} d g_{2} \cdots d g_{N} \delta\left(\sum_{i=1}^{N} g_{i}-L\right)
$$

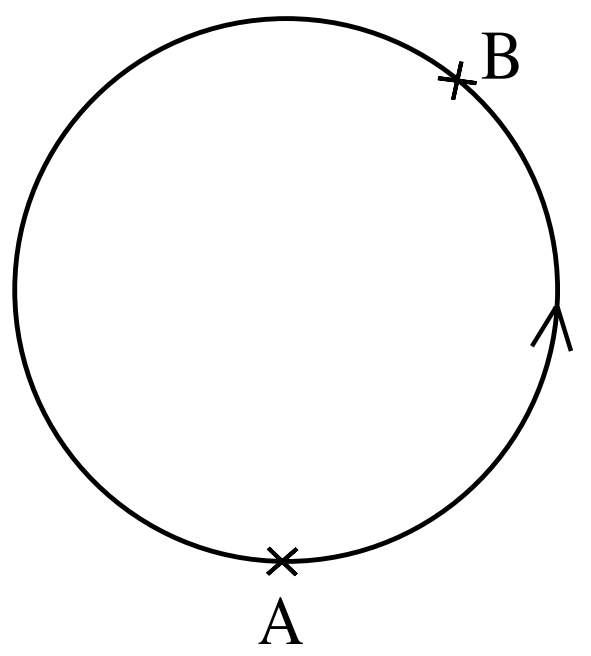

FIG. 1: Cycle of length $L$.

\section{Functions $F_{i}(r)$ For Free Fermions}

We now evaluate the function $D(r)$ of expression (3) using (13). Going from particle A to B, counterclockwise in the cycle of length $L$, as shown in Fig. 1, there may be $i$ other particles, with $i=0,1, \cdots,(N-2)$. Thus

$$
\rho D(r)=\rho\left[F_{0}(r)+F_{1}(r)+\cdots+F_{N-2}(r)\right],
$$

where

$$
\rho F_{i}(r) d r=\int \delta\left(\sum_{j=1}^{i+1} g_{j}-r\right) d r d P
$$

Thus

$$
\begin{aligned}
\rho F_{i}(r) & =(N-1) ! L \int|\Psi|^{2} \delta\left(\sum_{j=1}^{i+1} g_{j}-r\right) \\
& \times \delta\left(\sum_{j=i+2}^{N} g_{j}-(L-r)\right) d g_{1} d g_{2} \cdots d g_{N}
\end{aligned}
$$

where all $g_{j} \geq 0$.

Integrating over $d r$ we get, from (15),

$$
\int_{0}^{L} \rho F_{i}(r) d r=\int d P=1
$$

Thus by (14), $\int \rho D(r) d r=N-1$, confirming (4). Outside of the interval $(0, L)$ we define $F(r)$ by

$$
F(r)=0 \text { for } r<0 \text { and } r>L .
$$

Besides (17), $F_{i}(r)$ has also the following properties: (i) It is analytic except at $r=0$ and $r=L$, where $F(r)$ and its first derivative are both zero.

(ii) $F_{i}(r)=F_{N-2-i}(L-r)$.

It is obvious from (16) that $N F_{i}(r)$ is a function of $N$ and $r / L$. In Fig. 2 we plot this function vs $r / L$ for the case $N=5$.

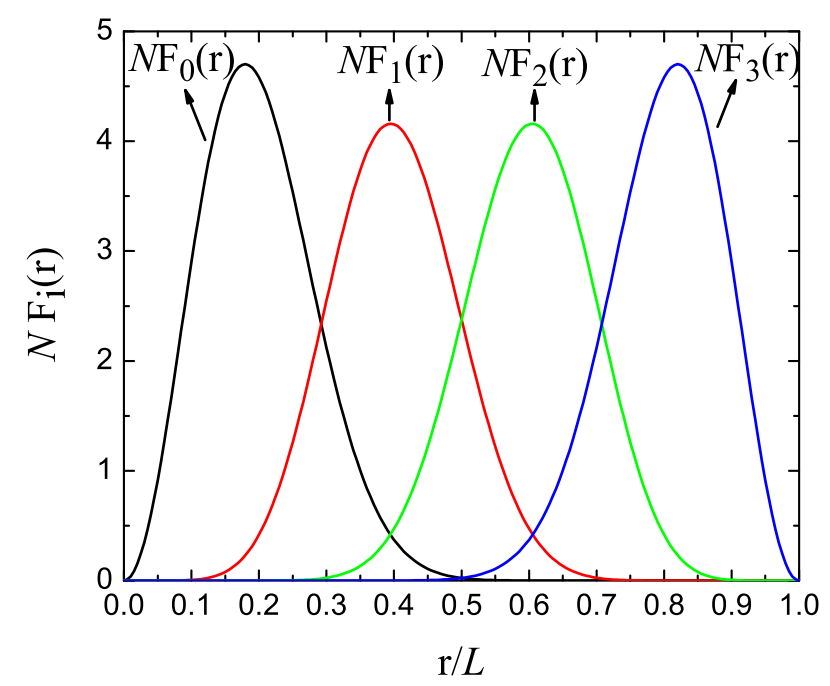

FIG. 2: $N F_{i}(r)$ against $r / L$ for $N=5$ and $i=0,1,2,3$. 

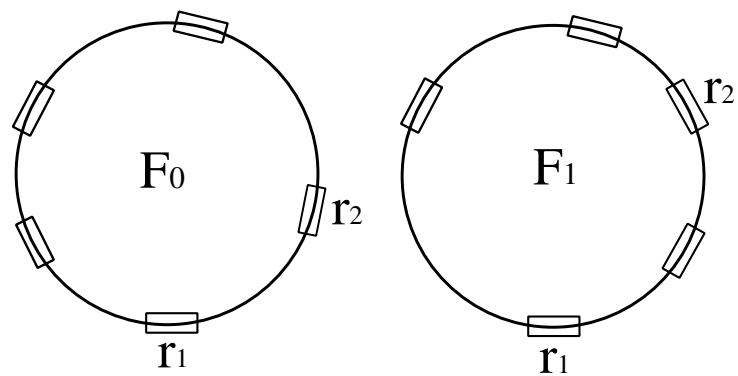

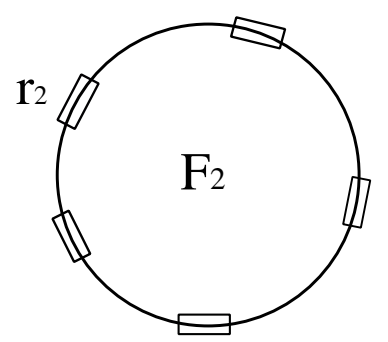

$r_{1}$

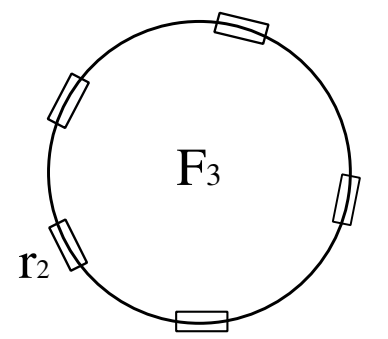

$\mathbf{r}_{1}$
FIG. 3: 4 possible configurations which contribute to the pair distribution function $D(r)$ for $N=5$. The ring represents the system and each block stands for a particle with diameter $a$.

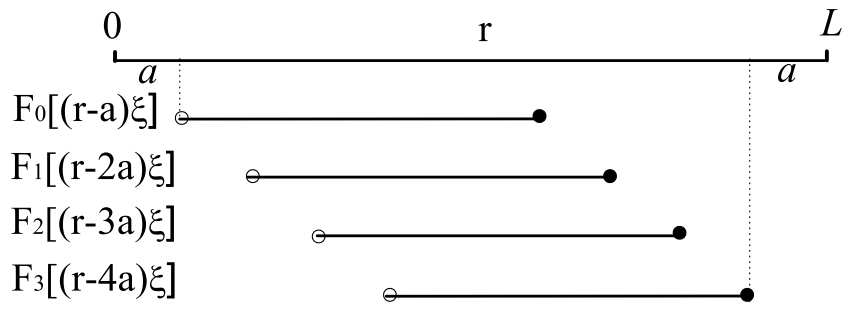

FIG. 4: Regions where $F_{i}(r)>0$ for $N=5$. Open circles are at $r=a, 2 a, 3 a, 4 a$. Closed circles are at $r=L-a, L-$ $2 a, L-3 a$ and $L-4 a$. These $2(N-1)=8$ circles are where $D(r)$ is singular. Each of the four regions has length $L-N a$.

\section{FERMIONS WITH $a>0$}

For fermions with $a>0$, the full coordinate space is again divided into $(N-1)$ ! regions, one of which, $R_{1}$, is defined by the cyclic condition (8) modulo $L$. We introduce gap variables in $R_{1}$ by equations similar to (9):

$$
\begin{aligned}
\quad g_{i}= & r_{i+1}-r_{i}-a, \quad i=1,2,3, \cdots,(N-1) \\
\text { and } \quad & g_{N}=r_{1}-r_{N}+L-a
\end{aligned}
$$

Obviously

$$
\sum g=L-N a
$$

The key point is that $\Psi$ in terms of the gap variables $\{g\}$ is still given by (10), but with

$$
f(g)=\sin (\pi g /(L-N a))
$$

and

$$
Q=\frac{1}{\sqrt{N !}}\left(\frac{1}{\sqrt{L-N a}}\right)^{N}\left[2^{N(N-1) / 2}\right] .
$$

Equation (12) now becomes

$$
|\Psi|^{2} d g_{1} d g_{2} \cdots d g_{N} \delta\left(\sum_{i=1}^{N} g_{i}-(L-N a)\right) L,
$$

and (13) beomes

$$
d P=(N-1) ! L|\Psi|^{2} d g_{1} \cdots d g_{N} \delta\left(\sum_{i=1}^{N} g_{i}-(L-N a)\right) .
$$

The function $D(r)$ is again, as in (14), a sum of $(N-$ 1) $F$ functions. To illustrate the reasoning we turn to Fig. 3 and Fig. 4 for the case $N=5$. We have

$$
\begin{aligned}
D(r) & =F_{0}[(r-a) \xi]+F_{1}[(r-2 a) \xi] \\
& +F_{2}[(r-3 a) \xi]+F_{3}[(r-4 a) \xi],
\end{aligned}
$$

where $\xi=L /(L-N a)$, and $F_{i}(r)$ is defined by (16) and (18).

Since $F_{i}(r)$ is nonzero only in the open interval $(0, L)$, for $N=5$,

$F_{0}[(r-a) \xi]$ is nonzero only for $a<r<L-4 a$,

$F_{1}[(r-2 a) \xi]$ is nonzero only for $2 a<r<L-3 a$,

$F_{2}[(r-3 a) \xi]$ is nonzero only for $3 a<r<L-2 a$,

$F_{3}[(r-4 a) \xi]$ is nonzero only for $4 a<r<L-a$.

In Fig. 4 we indicate the regions where $F_{i}[(r-(i+1) a) \xi]$ is nonzero for $i=0$ to 3 .

We present in Fig. 5 and Fig. 6 the function $D(r)$ for $N=5$, and for several values of $L / a$.

Returning now to the general case, we conclude

In the interval $(0, L), D(r)$ is analytic everywhere except at $(N-1)$ open circles at $r=a, 2 a, \cdots,(N-$ $1) a$, and at $(N-1)$ full circles at $r=L-a, L-$ $2 a, \cdots, L-(N-1) a$. At these singular points $D(r)$ is continuous and has a continuous first derivative.

\section{BOSONS WITH $a>0$}

For the one-dimensional hard sphere boson system, Girardeau [ㅁ, [] ] has shown a Bose-Fermi mapping theorem which maps the hardcore boson system to a spinless hardcore fermion system, for $a>0$.

$$
\Psi_{\mathrm{BE}}\left(x_{1}, x_{2}, \cdots, x_{N}\right)=\left|\Psi_{\mathrm{FD}}\left(x_{1}, x_{2}, \cdots, x_{N}\right)\right| .
$$

The pair distribution function is related to the square of the wave function. Thus for $a>0$

$$
D_{\mathrm{BE}}(r)=D_{\mathrm{FD}}(r) .
$$




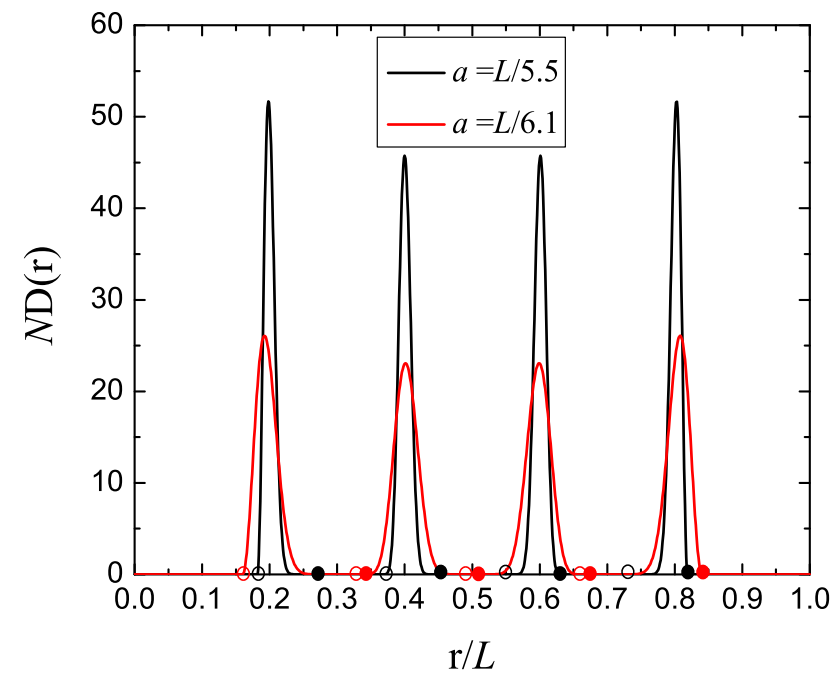

FIG. 5: (color online) The pair distribution function $D(r)$ as a function of distance $r$ for $N=5$. The $N-1=4$ open circles and the $N-1=4$ closed circles are where $D(r)$ is singular.

For case $a=L /(5+), N D(r)$ is four $\delta$ functions (not shown).

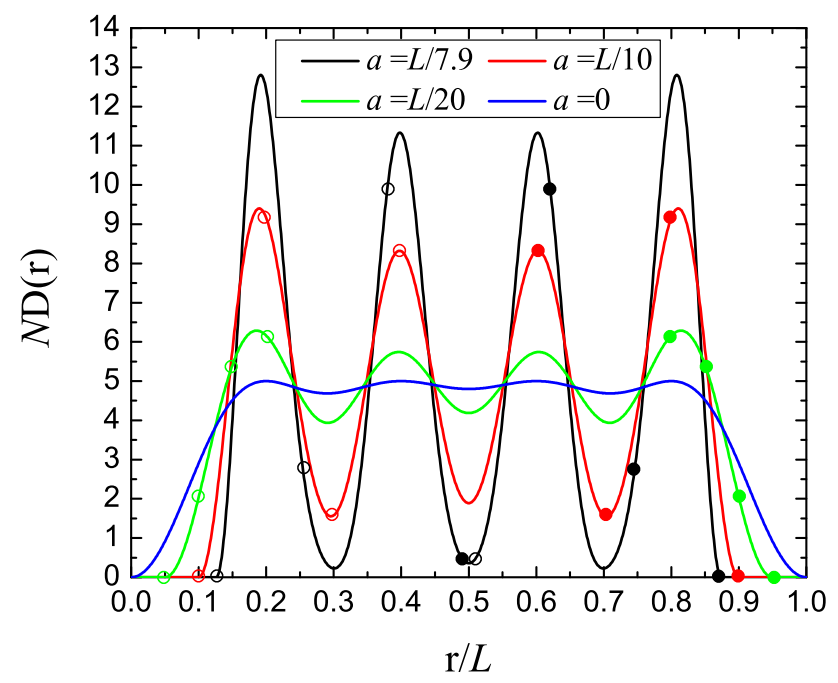

FIG. 6: (color online) The pair distribution function $D(r)$ as a function of distance $r$ for $N=5$.
[1] L. Tonks, Phys. Rev. 50, 955 (1936).

[2] E. Lieb and W. Liniger, Phys. Rev. 130, 1605 (1963).

[3] E. Lieb, Phys. Rev. 130, 1616 (1963).

[4] C. W. Ufford and E. P. Wigner, Phys. Rev. 61, 524 (1942).

[5] M. Girardeau, J. Math. Phys. 1, 516 (1960).

[6] M. D. Girardeau, Phys. Rev. 139, B500 (1965).

[7] Y. Castin et al., J. Mod. Opt. 47, 2671 (2000).

[8] D. M. Gangardt and G.V. Shlyapnikov, Phys. Rev. Lett. 90, 010401 (2003); New J. Phys. 5, 79 (2003).

[9] K.V. Kheruntsyan et al., Phys. Rev. Lett. 91, 040403 (2003); Phys. Rev. A 71, 053615 (2005).

[10] P. D. Drummond, P. Deuar, and K.V. Kheruntsyan, Phys. Rev. Lett. 92, 040405 (2004).

[11] G. E. Astrakharchik and S. Giorgini, J. Phys. B 39, S1 (2006); M. Cazalilla, ibid. 37, S1 (2004); J.-S. Caux and P. Calabrese, Phys. Rev. A 74, 031605(R) (2006).

[12] A. Cherny and J. Brand, Phys. Rev. A 73, 023612 (2006).

[13] A. G. Sykes et al., Phys. Rev. Lett. 100, 160406 (2008).

[14] B. B. Wei and C. N. Yang, cond-mat/0807.2081 (2008).

[15] C. N. Yang, Rev. Mod. Phys. 34, 694 (1962), §4. 